\title{
EUS-guided biliary drainage or enteroscopy-assisted ERCP in patients with surgical anatomy and biliary obstruction: an international comparative study
}

Authors

Institutions
Mouen A. Khashab ${ }^{1}$, Mohamad H. El Zein ${ }^{1}$, Kaveh Sharzehi ${ }^{2}$, Fernando P. Marson ${ }^{3}$, Oleh Haluszka ${ }^{2}$, Aaron J. Small ${ }^{4}$, Yousuke Nakai ${ }^{5}$, Do Hyun Park ${ }^{6}$, Rastislav Kunda ${ }^{7}$, Anthony Y. Teoh ${ }^{8}$, Irene Peñas ${ }^{9}$, Manuel Perez-Miranda ${ }^{9}$. Vivek Kumbhari ${ }^{1}$, Schalk Van der Merwe ${ }^{10}$, Everson L. Artifon ${ }^{3}$, Andrew S. Ross ${ }^{4}$

Institutions are listed at the end of article. submitted 18. April 2016 accepted after revision 13. June 2016

\section{Bibliography}

Dol http://dx.doi.org/

$10.1055 / \mathrm{s}-0042-110790$

Published online: 30.8.2016

Endoscopy International Open 2016; 04: E1322-E1327

(c) Georg Thieme Verlag KG

Stuttgart · New York

E-ISSN 2196-9736

\section{Corresponding author}

Mouen A. Khashab, MD

Associate Professor of Medicine

Director of Therapeutic

Endoscopy

Johns Hopkins Hospital

1800 Orleans St, Suite 7125 B

Baltimore, MD 21205

Phone: +1-443-287-1960

mkhasha1@jhmi.edu
License terms

(이 (1) $\ominus \circledast$
Background and study aims: How enteroscopyassisted ERCP (e-ERCP) and endoscopic ultrasound-guided biliary drainage (EUS-BD) compare in patients with surgically altered upper gastrointestinal anatomy is currently unknown. The aims of this study were to compare efficacy and safety of both techniques and study predictors of these outcomes.

Patients and methods: This was an international, multicenter comparative cohort study at 10 tertiary centers. Outcomes data included technical success (biliary access with cholangiography and stent placement [when indicated]), clinical success (resolution of biliary obstruction) and adverse events (AEs) (graded according to the ASGE lexicon).

Results: A total of 98 patients underwent EUS-BD $(n=49)$ or e-ERCP $(n=49)$. Technical success was achieved in 48 (98\%) patients in the EUS-BD group as compared to $32(65.3 \%)$ patients in the

\section{Introduction}

\section{$\nabla$}

Endoscopic ultrasound-guided biliary drainage (EUS-BD) is now an established technique mainly in patients who fail ERCP for various reasons, including gastric outlet obstruction, presence of duodenal stents, and surgically-altered upper gastrointestinal (gastrointestinal) anatomy [1 4]. Although multiple studies have shown excellent efficacy and good safety outcomes with EUSBD when performed by experts, its use specifically in patients with surgically-altered upper gastrointestinal anatomy has not been well studied. Enteroscopy-assisted ERCP (e-ERCP) is most commonly used for biliary access in patients with surgically-altered upper gastrointestinal anatomy with reported suboptimal clinical success rates between $48 \%$ and $70 \%$ [5]. In addition, e-ERCP is technically challenging, time consuming, and requires specialized equipment (e.g. balloon enteroscopy, accessories of appropriate length) [6].
e-ERCP group (OR 12.48, $P=0.001$ ). Clinical success was attained in $88 \%$ of patients in EUS-BD group as compared to $59.1 \%$ in the e-ERCP group (OR 2.83, $P=0.03$ ). Procedural time was significantly shorter in the EUS-BD group ( $55 \mathrm{~min}$ vs 95 min, $P<0.0001)$. AEs occurred more commonly in the EUS-BD group ( $20 \%$ vs. $4 \%, P=0.01$ ). However, the majority ( $90 \%$ ) of AEs were mild/moderate. Length of stay was significantly longer in the EUS-BD group (6.6 d vs. $2.4 \mathrm{~d}, P<0.0001$ ).

Conclusions: EUS-BD can be performed with a higher degree of clinical efficacy and shorter procedure time than e-ERCP in patients with surgically-altered upper gastrointestinal anatomy. Whether or not this approach should be first-line therapy in this patient population is highly dependent on the indication for the procedure, the patient's anatomy, and local practice and expertise.

Alternative methods of biliary access in these patients include laparoscopy-assisted ERCP and percutaneous biliary access [7]. Laparoscopy-assisted ERCP is only feasible in patients with Roux-en-Y gastric bypass anatomy, is relatively invasive, and requires coordination with a surgeon. Percutaneous biliary accesses is associated with non-trivial morbidity, is relatively invasive, requires repeated interventions and can negatively impact quality of life [1].

EUS-BD using transgastric access to the left liver lobe has been widely reported [ $8-10]$. This is particularly useful in patients with surgically-altered upper gastrointestinal anatomy as it avoids the need for enteroscopy. It is currently unknown how e-ERCP and EUS-BD compare in such patients. The primary aim of this study was to compare the clinical efficacy of both techniques in patients with surgically-altered upper gastrointestinal anatomy. Secondary goals were to compare both groups in terms of technical success, proce- 
dural time, and rate and severity of adverse events (AEs). In addition, we studied predictors of clinical success and occurrence of AEs.

\section{Patients and methods \\ $\nabla$}

This was an international, multicenter, retrospective comparative cohort study at 10 tertiary centers (3 United States, 3 European, 3 Asian and 1 South American). e-ERCP patients were recruited from 2 centers with published expertise on e-ERCP and EUS-BD patients from 8 centers with published expertise on EUS-BD. All procedures were performed between January 2008 and December 2014. All patients with surgically altered upper gastrointestinal anatomy who presented with benign or malignant biliary obstruction and subsequently underwent e-ERCP (2 centers) or EUS-BD (8 remaining centers) were included. Type of stent chosen was at the discretion of the treating endoscopist and was according to preference, expertise, cost, anatomy and availability. Relevant variables included demographics, type of surgical anatomy, enteroscopy technique, EUS-BD technique, and etiology of biliary obstruction. This retrospective study was approved by the Institutional Review Boards for Human Research and complied with Health Insurance Portability and Accountability Act (HIPAA) regulations at each participating institution.

\section{Definitions}

Technical success was defined as successful biliary access with cholangiography, stent placement in the desired location as determined endoscopically and/or radiographically, and/or stone removal, whenever indicated. Clinical success was defined as resolution of biliary drainage and reduction in serum total bilirubin by $50 \%$ at 1 week or less than $3 \mathrm{mg} / \mathrm{dL}$ at 2 weeks after the procedure in jaundiced patients. Procedure-related AEs were recorded and included: peritonitis, bile leak, cholangitis, bleeding, pancreatitis, intraperitoneal stent migration, subcapsular liver hematoma, pneumoperitoneum, perforation, retained sheared wire, and procedure-related death. All hospitalizations, procedures, and/or surgeries needed to treat procedure-related complications were tracked and recorded. Adverse events were graded according to the ASGE lexicon's severity grading system [11].

\section{Procedural techniques}

\section{EUS-guided biliary drainage}

Procedures were performed by interventional endoscopists with expertise in EUS, ERCP and interventional EUS. Procedures were performed as previously described [3]. The major anatomical modification in patients with surgical upper gastrointestinal anatomy was typically the absence of duodenal access. The majority of cases were typically performed using transgastric or transjejunal (in patients with total gastrectomy) access with hepatogastrostomy or hepatojejunostomy, respectively.

Rendezvous (RV) technique. A linear echoendoscope was used to achieve initial biliary access within a segment of dilated bile duct proximal to the site of obstruction. The tip of the echoendoscope was positioned in the gastric fundus. A 19-gauge or 22-gauge fine needle aspiration (FNA) needle (Echotip, Cook Medical, WinstonSalem, NC; or Expect 19 Flex, Boston Scientific, Natick, MA) was used to puncture a biliary segment with access confirmed by aspiration of bile and cholangiogram. Depending on the needle chosen either a 0.035-inch, 0.025-inch, or 0.018-inch guidewire was then advanced into the bile duct. The smaller 0.018-inch wires were exchanged for larger wires before stent placement. The echoendoscope and needle were angled to facilitate antegrade guidewire passage through the site of obstruction and across the papilla/anastomosis with coiling of the wire in the small bowel. The echoendoscope was then withdrawn leaving the guidewire in place. An endoscope was passed to the papilla/ anastomosis and a snare or biopsy forceps was used to grasp the guidewire and withdraw it through the endoscope with subsequent stent placement as per standard e-ERCP techniques.

Direct transluminal (TL) technique [hepatogastrostomy (HCS), hepatoduodenostomy (HDS), or hepatojejunostomy (HJS)]. In TL cases, the entire procedure was performed using the echoendoscope. After a biliary segment was accessed as described above, the biliary-enteric fistula was dilated with a catheter (e.g. 6 Fr cystotome; Cysto-Gastro set, Endo-flex, Voerde, Düsseldorf, Germany) or balloon, and a variety of devices were used to facilitate stent placement. These devices were selected based on the patient's anatomy and features of the obstructing stricture. Stent insertion was then performed leaving at least $3 \mathrm{~cm}$ of stent with the gastrointestinal lumen to avoid intraperitoneal stent migration.

Antegrade stenting (AGS). The dilated biliary ductal segment was punctured with an FNA needle and contrast was then injected through the needle to provide a cholangiogram. A hydrophilic guidewire was advanced through the needle and manipulated across the ampulla/anastomosis. The FNA needle was then removed, and the tract was created using a 6 Fr cystotome (CystoGastro set, Endo-flex, Voerde, Düsseldorf, Germany) or dilated as described above. To facilitate antegrade stent placement, strictures, when present, were dilated before advancing the stent through the therapeutic channel of the echoendoscope over the guide wire. The stent was then deployed either across the stricture transpapillary or trans-anastomotic in patients with biliary-enteric anastomoses.

\section{Enteroscopy-assisted ERCP}

Procedures were performed using single-balloon enteroscope (SBE, Olympus Optical, Tokyo, Japan), double balloon-enteroscope (DBE, Fujifilm Medical, Tokyo, Japan) or pediatric colonoscope (Olympus). e-ERCP using SBE and DBE were performed as described before. Advancement through the small bowel was achieved with a series of cycles using a push-and-pull technique. A pediatric colonoscope was occasionally used at the discretion of the endoscopist. A transparent cap was attached to the tip of the endoscope according to the preference of the operator. Accessories compatible with enteroscopes were used during ERCP. Sphincterotomy, sphincteroplasty, stricture dilation, stone removal and/or stent placement were performed as required.

\section{Statistical analysis \\ $\nabla$}

Results are reported as mean \pm standard deviation (SD) or median (range) for quantitative variables and percentages for categorical variables. Outcomes between EUS-BD and e-ERCP groups were compared using the Student's $t$ test (or the Wilcoxon rank-sum test if required) for continuous variables and the chi-square test (or Fisher's exact test if required) for categorical variables. Univariable analysis was performed by using logistic regression to assess factors (RY anatomy native papilla, age, gender, baseline bilirubin, and technique, EUSBD vs e-ERCP) associated with clinical success and AEs. Multivariable regression analysis was performed taking into account the variables, native papilla, RY anat- 
Table 1 Baseline patient and procedural characteristics of 98 patients.

\begin{tabular}{|l|c|}
\hline Variable & $\begin{array}{l}\text { Total } \\
(\mathbf{n = 9 8 )}\end{array}$ \\
\hline Age (yrs), mean \pm SD & $58 \pm 13.9$ \\
\hline Female, $\mathrm{n}(\%)$ & $66(67.3)$ \\
\hline Malignant indication, $\mathrm{n}(\%)$ & $35(35.7)$ \\
\hline RY anatomy, $\mathrm{n}(\%)$ & $69(70)$ \\
\hline Native papilla, $\mathrm{n}(\%)$ & $66(67)$ \\
\hline Baseline bilirubin, mean \pm SD & $5.1 \pm 6.2$ \\
\hline Stent material, $\mathrm{n}(\%)$ & \\
\hline Metal & $40(40.8)$ \\
\hline Plastic & $18(16.4)$ \\
\hline No stent & $40(40.8)$ \\
\hline Procedure duration (min), mean \pm SD & $77 \pm 41$ \\
\hline Length of hospital stay (day), median (range) & $3(0-30)$ \\
\hline Technical success, $\mathrm{n}(\%)$ & $80(81.6)$ \\
\hline Clinical success, $\mathrm{n}(\%)$ & $72(73.4)$ \\
\hline Adverse events, $\mathrm{n}(\%)$ & $12(12.2)$ \\
\hline All & $4(4)$ \\
\hline Mild & $7(7.1)$ \\
\hline Moderate & $1(1)$ \\
\hline Severe & 0 \\
\hline Death & \\
\hline
\end{tabular}

omy and procedural technique. Statistical significance was based on two-sided design-based tests evaluated at a $=0.05$. Statistical analysis was performed using SPSS version 16 (SPSS Inc, Chicago, IL, USA).

\section{Results}

$\nabla$

A total of 98 patients (mean age $58 \mathrm{yr}$, female $67 \%$ ) underwent either EUS-BD $(n=49)$ or e-ERCP $(n=49)$ for management of suspected biliary obstruction during the study period ( $\square$ Table1). Etiology of biliary obstruction was malignant in nature in
35 (36\%) patients and benign in the remainder 63 (64\%). Type of surgical upper gastrointestinal anatomy was pancreaticoduodenectomy (Whipple procedure) in 15, Billroth II 12, Roux-en-y hepaticojejunostomy 17 , Roux-en-y gastric bypass (RYGB) 52 , and total gastrectomy with esophagojejunostomy 2. Roux-en-Y (RY) anatomy was encountered in a total of $69(70 \%)$ patients. Native papilla was encountered in 66 (67\%) cases while an enteric-biliary anastomosis was seen in 32 (32\%). The mean preprocedural bilirubin was $5.1 \mathrm{mg} / \mathrm{dL}(0.2-25)$ ( Table 1$)$.

Among 49 patients who underwent EUS-BD, malignant biliary obstruction accounted for the majority of cases ( $n=33,67 \%)$. RY reconstruction was encountered in $24(49 \%)$ patients. A native papilla was seen in 24 (49\%) patients while an enteric-biliary anastomosis was seen in the remainder $25(51 \%)$ patients. The mean preprocedural bilirubin was $8.8 \pm 6.6 \mathrm{mg} / \mathrm{dL}$. EUS-BD technique used was HGS in 33, AGS in 10, HJS in 3, RV in 2, and HDS in 1.

Among 49 patients who underwent e-ERCP, benign biliary obstruction accounted for the majority of cases $(n=47,96 \%)$. RY reconstruction was encountered in 45 (92\%) patients. A native papilla was seen in 42 (85.7\%) patients while an enteric-biliary anastomosis was seen in the remainder $7(14.3 \%)$ patients. The mean preprocedural bilirubin was $1.48 \pm 2.5 \mathrm{mg} / \mathrm{dL}$. e-ERCP technique used was DBE-ERCP in 42, SBE-ERCP 5, and colonoscopeERCP in 2.

There was a higher frequency of malignant obstruction in the EUS-BD group as compared to the e-ERCP group $(P<0.0001)$, but higher frequency of RY anatomy $(P<0.0001)$ and native ampulla $(P<0.0001)$ in the e-ERCP group $($ Table 2$)$. Technical success was achieved in 48 (98\%) patients in the EUS-BD group as compared to $32(65.3 \%)$ patients in the e-ERCP group (OR 12.48, 95\% CI 2.69-57.78, $P=0.001)$. Among the 17 technical failures in the e-ERCP group, 11 resulted from failure to reach the ampulla or enteric-biliary anastomosis and 6 were due to failed cannulation. Clinical success was attained in $88 \%$ of patients in EUS-BD group as compared to $59.1 \%$ in the e-ERCP group (OR 2.83, 95\% CI $1.10-$ $7.31, P=0.03$ ). Procedural time was significantly shorter in the

\begin{tabular}{|c|c|c|c|}
\hline Variable & $\begin{array}{l}\text { EUS-BD } \\
(n=49)\end{array}$ & $\begin{array}{l}\text { e-ERCP } \\
(n=49)\end{array}$ & $P$ value \\
\hline Age $(y r)$, mean \pm SD & $62.48 \pm 14.9$ & $53.6 \pm 11.46$ & $P<0.001$ \\
\hline Female, n (\%) & $22(44.8)$ & $44(89.7)$ & $P<0.001$ \\
\hline Malignant indication, $\mathrm{n}(\%)$ & $33(67.3)$ & $2(4)$ & $P<0.001$ \\
\hline RY anatomy, n (\%) & $24(49)$ & $45(92)$ & $P<0.001$ \\
\hline Native papilla, n (\%) & $24(49)$ & $42(85.7)$ & $P<0.001$ \\
\hline Technique, n (\%) & $\begin{array}{l}\text { HG } 33(67.4) \\
\text { AS } 10(20.5) \\
\text { RV } 2(4) \\
\text { HJ } 3(6.1) \\
\text { HD } 1(2)\end{array}$ & $\begin{array}{l}\text { SBE-ERCP } 5 \text { (10.2) } \\
\text { DBE-ERCP } 42(85.7) \\
\text { CS-ERCP } 2(4.1)\end{array}$ & NA \\
\hline Ampulla/biliary anastomosis reached & NA & $\begin{array}{l}\text { Yes } 38 \\
\text { No } 11\end{array}$ & NA \\
\hline Baseline bilirubin, mean \pm SD & $8.8 \pm 6.6$ & $1.4 \pm 2.5$ & $P<0.001$ \\
\hline Procedure duration (min), mean \pm SD & $55 \pm 36$ & $95.7 \pm 38.5$ & $P<0.001$ \\
\hline Length of hospital stay (day), median(range) & $3(1-30)$ & $1(0-15)$ & $P<0.001$ \\
\hline Technical success, $\mathrm{n}(\%)$ & $48(98)$ & $32(65.3)$ & $P=0.001$ \\
\hline Clinical success, n (\%) & $43(88)$ & $29(59.1)$ & $P=0.03$ \\
\hline \multicolumn{4}{|l|}{ Adverse events, n (\%) } \\
\hline All & $10(20.4)$ & $2(4.1)$ & $P=0.03$ \\
\hline Mild & $3(6.1)$ & $1(2)$ & $P=0.61$ \\
\hline Moderate & $6(12.2)$ & $1(2)$ & $P=0.06$ \\
\hline Severe & $1(2)$ & $0(0)$ & $P=0.5$ \\
\hline Death & $0(0)$ & $0(0)$ & NA \\
\hline
\end{tabular}

Table 2 Comparison of characteristics and outcomes between EUS-BD and e-ERCP groups. 
Table 3 Univariate analysis of factors associated with clinical success and AEs.

\begin{tabular}{|llll|}
\hline & OR & $95 \% \mathbf{C l}$ & $\boldsymbol{P}$ value \\
\hline Clinical success & & & \\
\hline EUS-BD vs ERCP & 2.83 & $1.10-7.31$ & 0.03 \\
\hline RY anatomy & 0.829 & $0.30-2.28$ & 0.72 \\
\hline Native papilla & 1.035 & $0.40-2.70$ & 0.94 \\
\hline Age & 1.02 & $0.99-1.06$ & 0.13 \\
\hline Female & 4.5 & $1.2-16.7$ & 0.02 \\
\hline Baseline bilirubin & 1.0 & $0.95-1.1$ & 0.47 \\
\hline Adverse events & & & \\
\hline EUS-BD vs ERCP & 6.03 & $1.25-29.15$ & 0.03 \\
\hline RYanatomy & 0.51 & $0.15-1.77$ & 0.29 \\
\hline Native papilla & 1.69 & $0.43-6.71$ & 0.45 \\
\hline Age & 1.0 & $0.96-1.06$ & 0.54 \\
\hline Female & 2.4 & $0.64-9.42$ & 0.18 \\
\hline Baseline bilirubin & 1.0 & $0.94-1.13$ & 0.48 \\
\hline
\end{tabular}

EUS-BD = endoscopic ultrasound-guided biliary drainage; $E R C P=$ endoscopic retrograde cholangiopancreatography

EUS-BD group as compared to the e-ERCP group (55 minutes vs 95 minutes, respectively, $P<0.0001$ ).

The rate of AEs was significantly higher in the EUS-BD group ( $20 \%$ vs. $4 \%$, OR 5.3, $95 \%$ CI $0.03-0.9, P=0.01$ ); however, the majority (90\%) of AEs were mild/moderate in nature. There was 1 severe AE (sepsis) in the EUS-BD group. Nine other complications were encountered in patients who underwent EUS-BD ( 3 cholangitis, 2 sepsis, 2 bleeding, 1 pneumoperitoneum, and 1 retained sheared wire). Only 2 complications occurred in patients who underwent e-ERCP, one mild and one moderate pancreatitis. The length of hospital stay was significantly longer in the EUS-BD group (6.6 days vs. 2.4 days, $P=<0.0001$ ) ( $\bullet$ Table 2 ).

On univariate analysis, EUS-BD was associated with increased rate of clinical success (OR 2.83, 95\% CI 1.10-7.31, $P=0.03$ ) and AEs (OR 6.03, 95\% CI 1.25-29.15, $P=0.03$ ) ( Table3). Multivariable analysis also showed that EUS-BD was associated with increased rate of clinical success (OR 4.31, 95\% CI 1.28-14.5, $P=$ 0.02 ) and AEs (OR 8.74, 95\% CI 1.54-49.72,P=0.01) ( Table 4). Subgroup analysis showed that EUS-BD using HGS technique was equally effective and safe compared to the other EUS-BD techniques (all $P>0.05$ ). Similarly, e-ERCP performed using DBEERCP was equally effective and safe compared to the other eERCP techniques (all $P>0.05$ ).

\section{Discussion}

$\nabla$

Enteroscopy-assisted ERCP has been utilized extensively for biliary access and interventions in patients with surgically altered upper gastrointestinal anatomy, but with suboptimal results $[5,12]$. EUS is an ideal modality for visualization and access of the biliary tree in such patients as the left lobe of the liver is readily seen transgastrically. The current study is the first to compare e-ERCP and EUS-BD in such patients who present with biliary obstruction. A total of 98 patients underwent either EUS-BD $(n=49)$ or e-ERCP $(n=49)$. Rates of technical success ( $98 \%$ vs $65 \%$ ) and clinical success ( $88 \%$ vs $60 \%$ ) were both significantly higher in patients who underwent EUS-BD, although anatomy and procedural indications were significantly different between groups. EUS$\mathrm{BD}$ procedures were significantly less time consuming with an average of 40 minutes saved per procedure. The rate of AEs was significantly higher in the EUS-BD group, although the vast majority were not severe. The length of stay was significantly longer in the EUS-BD group, but that could be due to underlying pathology (predominantly malignant) rather than the procedure technique itself.

A number of studies have described the use of e-ERCP in patients with surgically-altered upper gastrointestinal anatomy. Shah and colleagues presented a multicenter US experience with e-ERCP in patients with surgically altered pancreaticobiliary anatomy [12]. A total of 129 patients had 180 e-ERCP with RYGB $(n=63)$ and RY hepaticojejunostomy $(n=45)$ as the most commonly encountered surgical anatomy. Successful ERCP was achieved in $63 \%$ of patients and was similar between RYGB and other long-limb surgical bypass patients. AEs occurred in $12 \%$ of patients. Skinner and colleagues performed a systematic review of e-ERCP performed in patients with surgical upper gastrointestinal anatomy [5]. A total of 945 procedures in 679 patients who had a variety of post-surgical upper gastrointestinal anatomical configurations were included. Overall ERCP success was 74\%. Among patients with RYGB anatomy, ERCP success was achieved in 70\% of cases. In patients who had undergone a RY with either a pancreaticoduodenectomy or hepaticojejunostomy, ERCP success was $76 \%$. In patients with Billroth II anatomy, the rate of success with ERCP was $90 \%$. The overall rate of AEs was 3.4\%. Outcomes of eERCP in the current study are in line with these published results, which illustrates moderate success and excellent safety of e-ERCP in such patients.

Dedicated studies on EUS-BD in patients with altered anatomy are scarce. Siripun et al. reported a systematic review of all reported EUS-BD in such patients [13]. Seventy four cases were included for analysis. The pooled technical success, clinical success, and $\mathrm{AE}$ rates were $89 \%, 91 \%$, and $17.5 \%$, respectively. Reported

\begin{tabular}{|c|c|c|c|c|c|c|}
\hline & \multicolumn{3}{|c|}{ Unadjusted } & \multicolumn{3}{|c|}{ Adjusted } \\
\hline & OR & $95 \% \mathrm{Cl}$ & $P$ value & OR & $95 \% \mathrm{Cl}$ & $P$ value \\
\hline EUS-BD vs ERCP & 2.83 & $1.10-7.31$ & 0.03 & 4.31 & $1.28-14.54$ & 0.02 \\
\hline RY anatomy & 0.829 & $0.30-2.28$ & 0.72 & 1.48 & $0.41-5.35$ & 0.54 \\
\hline Native papilla & 1.035 & $0.4-2.70$ & 0.94 & 1.75 & $0.54-5.61$ & 0.34 \\
\hline \multicolumn{7}{|l|}{ Adverse events } \\
\hline EUS-BD vs ERCP & 6.03 & $1.25-29.15$ & 0.03 & 8.74 & $1.54-49.72$ & 0.01 \\
\hline RY & 0.51 & $0.15-1.77$ & 0.29 & 0.87 & $0.21-3.57$ & 0.84 \\
\hline Native papilla & 1.69 & $0.43-6.71$ & 0.45 & 3.63 & $0.82-15.92$ & 0.08 \\
\hline
\end{tabular}

EUS-BD = endoscopic ultrasound-guided biliary drainage; $E R C P=$ endoscopic retrograde cholangiopancreatography 
complications included pancreatitis, abdominal pain, hematoma, cholangitis, minor bleeding, and peritoneal stent migration, with no mortalities. Itoi et al. reported on EUS- transhepatic antegrade stone removal in patients with surgically altered anatomy with excellent outcomes [14]. These results are similar to the reported outcomes of > EUS-BD in general [15]. In addition, these outcomes are in agreement with results from the current study. e-ERCP offers some advantages over EUS-BD. It is an established procedure that is widely available and practiced. Dedicated ERCP devices compatible for use through enteroscopes are available from multiple manufacturers. Furthermore, the procedure is very safe with rare severe AEs and is associated with moderate clinical success rate. Management of choledocholithiasis using eERCP remains the main technique of choice as the EUS-guided approach remains challenging. On the other hand, EUS-BD also has multiple benefits over e-ERCP. The biliary system is readily accessible transgastrically, which avoids the need for the time consuming navigation deep into the small bowel. EUS-BD is therefore a more efficient procedure as was shown in the current study. Success of EUS-BD is not affected by long surgical limbs and angulations (e.g. at the jejunojenunostomy). This is reflected by a higher clinical success of EUS-BD as was also shown in the current study. Lastly, EUS-BD allows placement of the larger self-expandable metallic stents when needed, which is associated with improved drainage and longer patency rates as compared to plastic stents.

It is important to highlight other established endoscopic methods that can also be employed in patients with surgically-altered upper gastrointestinal anatomy, including ERCP through a gastrostomy tract and laparoscopy-assisted ERCP in patients with RYGB anatomy. A study comparing outcomes of ERCP via gastrostomy and eERCP for patients with previous RYGB found that ERCP via gastrostomy was successful in $95 \%$ of cases, whereas e-ERCP was successful in $63 \%$ of cases. However, the rate of AEs was higher with ERCP via gastrostomy (14.5\%) compared with e-ERCP (14.5\% vs $3.1 \%, P=0.02$ ). The authors concluded that ERCP via gastrostomy has a higher success rate than e-ERCP but is hindered by the gastrostomy maturation delay and a higher morbidity [16]. Another study comparing laparoscopy-assisted ERCP ( $\mathrm{n}=$ $24)$ with eERCP $(n=32)$ in patients with RYGB showed $100 \%$ ERCP success rate in the laparoscopy-assisted ERCP group and 59\% in the e-ERCP group [7]. In patients with a Roux limb plus ligament of Treitz to jejunojejunal anastomosis limb length of more than $150 \mathrm{~cm}$, successful e-ERCP was only achieved in $25 \%$ of patients. Complications were minor and infrequent in both groups. A cost analysis showed that patients with a Roux limb plus ligament of Treitz to jejunojejunal anastomosis limb length of less than $150 \mathrm{~cm}$ should be offered e-ERCP first, and those with longer limbs should proceed directly to laparoscopy- assisted ERCP [7]. There are significant limitations to the current study. First, all procedures were performed by experts in pancreaticobiliary endoscopy, therefore, results may not be generalizable. It is a retrospective study with inherent limitations due to the study design. Patients from each group were selected from different centers according to expertise and center-effect was not accounted for. Finally, the baseline characteristics of patients were not similar between the two groups. The majority of patients undergoing e-ERCP had benign disease and RYGB anatomy as opposed to the EUS-BD group where the majority of patients had malignant indications for biliary drainage and non-RYGB surgically altered upper gastrointestinal anatomy. As such, the outcome of any direct comparison between the 2 techniques is subject to signifi- cant selection bias. Randomized studies are need for optimal comparison but will be extremely difficult to perform as stratification for long limbs, dilated biliary system and other variables will be necessary.

These data suggest that EUS-BD can be performed with a high degree of clinical efficacy and moderate safety in patients with surgically-altered upper gastrointestinal anatomy. Whether this approach should be first-line therapy in this patient population is highly dependent on the indication for the procedure, the patient's anatomy and finally, local practice bias and expertise in either e-ERCP or EUS-BD. We believe that EUS-BD is still best offered to patients after a failed initial e-ERCP; however, in patients with malignant biliary obstruction and expected long surgical limbs, the rate of technical failure of e-ERCP is high and EUS-BD can be offered as a first-line modality if appropriate expertise is available.

Competing interests: Dr. Khashab is a consultant for Boston Scientific and Xlumena Manuel Perez-Miranda is a consultant for Boston Scientific. Dr. Kunda is a consultant for Boston Scientific, Olympus and Xlumena.

Institutions

${ }^{1}$ Division of Gastroenterology and Hepatology, The Johns Hopkins Medical Institutions, Baltimore, MD, United States

${ }^{2}$ Division of Gastroenterology and Hepatology, Temple University Hospital, Philadelphia, PA, United States

${ }^{3}$ Hospital Ana Costa. Santos, Brazil

${ }^{4}$ Division of Hepatology and Gastroenterology, Virginia Mason Medical Center, Seattle, WA, United States

Department of Gastroenterology, Graduate School of Medicine, The University of Tokyo, Tokyo, Japan

${ }^{6}$ Department of Internal Medicine, University of Ulsan College of Medicine, Asan Medical Center, Seoul, South Korea

Department of Surgical Gastroenterology, Aarhus University Hospital, Aarhus, Denmark

${ }^{8}$ Department of Surgery, The Chinese University of Hong Kong, Hong Kong, China

${ }^{9}$ Hospital Universitario Rio Hortega, Valladolid University, Valladolid, Spain

${ }^{10}$ Department of Hepatology, KU leuven, Leuven, Belgium

\section{References}

1 Khashab MA, Valeshabad AK, Afghani $E$ et al. A comparative evaluation of EUS-guided biliary drainage and percutaneous drainage in patients with distal malignant biliary obstruction and failed ERCP. Dig Dis Sci 2015; 60: 557-565

2 Khashab MA, Fujii LL, Baron TH et al. EUS-guided biliary drainage for patients with malignant biliary obstruction with an indwelling duodenal stent (with videos). Gastrointest Endosc 2012; 76: 209-213

3 Khashab MA, Levy MJ, Itoi T et al. EUS-guided biliary drainage. Gastrointest Endosc 2015; 82: $993-1001$

4 Perez-Miranda M, De la Serna-Higuera C. EUS access to the biliary tree. Curr Gastroenterol Rep 2013; 15: 349

5 Skinner M, Popa D, Neumann $H$ et al. ERCP with the overtube-assisted enteroscopy technique: a systematic review. Endoscopy 2014; 46: $560-572$

6 Khashab MA, Okolo PI3rd. Accessing the pancreatobiliary limb and ERCP in the bariatric patient. Gastrointest Endosc Clin N Am 2011; 21: $305-313$

7 Schreiner MA, Chang L, Gluck M et al. Laparoscopy-assisted versus balloon enteroscopy-assisted ERCP in bariatric post-Roux-en-Y gastric bypass patients. Gastrointest Endosc 2012; 75: 748-756

8 Khashab MA, Kumbhari V, Kalloo AN et al. EUS-guided biliary drainage by using a hepatogastrostomy approach. Gastrointest Endosc 2013; 78: 675

9 Artifon EL, Marson FP, Gaidhane M et al. Hepaticogastrostomy or choledochoduodenostomy for distal malignant biliary obstruction after failed ERCP: is there any difference? Gastrointest Endosc 2015; 81: 950-959 
10 Shah JN, Marson F, Weilert F et al. Single-operator, single-session EUSguided anterograde cholangiopancreatography in failed ERCP or inaccessible papilla. Gastrointest Endosc 2012; 75: 56-64

11 Cotton PB, Eisen GM, Aabakken $L$ et al. A lexicon for endoscopic AEs: report of an ASGE workshop. Gastrointest Endosc 2010; 71: 446 - 454

12 Shah RJ, Smolkin M, Yen R et al. A multicenter, U.S. experience of singleballoon, double-balloon, and rotational overtube-assisted enteroscopy ERCP in patients with surgically altered pancreaticobiliary anatomy (with video). Gastrointest Endosc 2013; 77: 593-600

13 Siripun A, Sripongpun P, Ovartlarnporn B. Endoscopic ultrasound-guided biliary intervention in patients with surgically altered anatomy. World J Gastrointest Endosc 2015; 7: 283-289
14 Itoi T, Sofuni A, Tsuchiya $T$ et al. Endoscopic ultrasonography-guided transhepatic antegrade stone removal in patients with surgically altered anatomy: case series and technical review (with videos). J Hepatobiliary Pancreat Sci 2014; 21: E86-93

15 Khashab MA, Dewitt J. EUS-guided biliary drainage: is it ready for prime time? Yes! Gastrointest Endosc 2013; 78: $102-105$

16 Choi EK, Chiorean MV, Cote GA et al. ERCP via gastrostomy vs. double balloon enteroscopy in patients with prior bariatric Roux-en-Y gastric bypass surgery. Surg Endosc 2013; 27: 2894-2899 